\title{
Molecular Imaging with ${ }^{11} \mathrm{C}$-PD153035 PET/CT Predicts Survival in Non-Small Cell Lung Cancer Treated with EGFR-TKI: A Pilot Study
}

\author{
Xue Meng ${ }^{1}$, Billy W. Loo Jr. ${ }^{2}$, Li Ma ${ }^{3}$, James D. Murphy ${ }^{2}$, Xindong Sun ${ }^{1}$, and Jinming $\mathrm{Yu}^{1}$ \\ ${ }^{I}$ Department of Radiation Oncology, Shandong Cancer Hospital, Jinan, Shandong, China; ${ }^{2}$ Department of Radiation Oncology, \\ Stanford University and Cancer Institute, Stanford, California; and ${ }^{3}$ PET/CT Center, Shandong Cancer Hospital, Jinan, Shandong, \\ China
}

\begin{abstract}
Outcomes are suboptimal when molecularly targeted therapies are used in patient populations unselected for the molecular target. This pilot study examines the correlation of PET using ${ }^{11} \mathrm{C}$-labeled 4-N-(3-bromoanilino)-6,7-dimethoxyquinazoline (11C-PD153035), an imaging biomarker of epidermal growth factor receptor (EGFR), with outcomes in patients with nonsmall cell lung cancer (NSCLC) treated with the EGFR tyrosine kinase inhibitor erlotinib. Methods: Patients with advanced chemotherapy-refractory NSCLC were prospectively enrolled on a trial of erlotinib at a dose of $150 \mathrm{mg}$ daily and imaged by ${ }^{11} \mathrm{C}-\mathrm{PD} 153035$ PET/CT at baseline, after 1-2 wk, and after $6 \mathrm{wk}$ from the start of treatment. Overall survival and progressionfree survival (OS and PFS, respectively) times were correlated with the ${ }^{11} \mathrm{C}-\mathrm{PD} 153035$ standardized uptake value (SUV) at each of the imaging times. Results: Twenty-one patients were enrolled. Follow-up to progression was complete in all patients and to death in 18 of 21 . By Cox regression analysis, baseline maximum SUV correlated strongly with OS and PFS (hazard ratio $=0.40, P=0.002$, and hazard ratio $=0.044, P<0.001$, respectively) independent of histology. Patients with higher maximum SUV ( $\geq$ median) survived more than twice as long as patients with lower maximum SUV (median OS $=11.4$ vs. $4.6 \mathrm{mo}, P=0.002$; PFS $=4.4$ vs. $1.8 \mathrm{mo}, P<0.001$ ). However, ${ }^{11} \mathrm{C}-\mathrm{PD} 153035$ uptake on follow-up scans was less well correlated with survival. Conclusion: Our preliminary results suggest ${ }^{11} \mathrm{C}-\mathrm{PD} 153035 \mathrm{PET} / \mathrm{CT}$ may be a noninvasive and rapid method for identifying patients with refractory advanced NSCLC of adenocarcinoma or squamous histology likely to respond to the EGFR tyrosine kinase inhibitor but not for monitoring treatment response.
\end{abstract}

Key Words: PD153035; PET; EGFR; molecular imaging; NSCLC

J Nucl Med 2011; 52:1573-1579

DOI: 10.2967/jnumed.111.092874

\footnotetext{
Received May 9, 2011; revision accepted Jul. 29, 2011.

For correspondence or reprints contact: Jinming Yu, Department of Radiation Oncology, Key Laboratory of Radiation Oncology of Shandong

Province, Shandong Cancer Hospital, 440 Jiyan Rd., Jinan, Shandong

Province 250117, China.

E-mail: f1paul@126.com

Published online Sep. 8, 2011.

COPYRIGHT @ 2011 by the Society of Nuclear Medicine, Inc.
}

$\mathbf{N}$ on-small cell lung cancer (NSCLC) is the leading cause of cancer deaths in the world (1). Chemotherapy, the mainstay of treatment in advanced disease, is only modestly effective $(2,3)$. In recent years, the epidermal growth factor receptor (EGFR) pathway has been found to be an important driver of tumor progression in various cancers, including NSCLC, and thus an attractive therapeutic target. The 2 most commonly used tyrosine kinase inhibitors (TKIs) targeting EGFR are gefitinib (Tarceva; Genentech/ OSI Pharmaceuticals) and erlotinib (Iressa; AstraZeneca).

When using molecularly targeted drugs, identifying the presence of the molecular target is crucial to achieving optimal outcomes. For example, in the first-line setting, the Asian Iressa Pan-Asia Study demonstrated that even in patients selected by clinical features for responsiveness to gefitinib, patients with wild-type EGFR had significantly worse survival with gefitinib than with conventional chemotherapy, whereas the reverse was true in patients with activating mutations in EGFR (4). However, in the setting of second-line therapy and beyond, the National Cancer Institute of Canada (NCIC) Clinical Trials Group BR.21 trial demonstrated superiority of erlotinib over placebo (5) and the INTEREST trial demonstrated the noninferiority of gefitinib, compared with docetaxel (6), both in unselected chemotherapy-refractory patient populations. Interestingly, neither study found EGFR mutations to be significantly predictive of improved survival with EGFRTKIs $(7,8)$, indicating the need for additional predictors of outcomes for optimal patient selection.

${ }^{11} \mathrm{C}$-labeled 4- $\mathrm{N}$-(3-bromoanilino)-6,7-dimethoxyquinazoline ( $\left.{ }^{11} \mathrm{C}-\mathrm{PD} 153035\right)$, a positron-emitting analog of the EGFR-TKI PD153035, was developed as a noninvasive imaging biomarker for tumor EGFR status using PET (9). We previously demonstrated preclinically that ${ }^{11} \mathrm{C}-\mathrm{PD} 153035$ tumor uptake correlates with EGFR expression (10) and characterized its biodistribution in healthy human volunteers as well as demonstrated tumor uptake in patients with NSCLC (11). Therefore, ${ }^{11} \mathrm{C}-\mathrm{PD} 153035 \mathrm{PET} / \mathrm{CT}$ has the potential to serve as an imaging biomarker for predicting which patients will benefit most from EGFR-TKI treatment. 
In this study, we sought to determine whether ${ }^{11} \mathrm{C}$ PD153035 PET/CT correlated with outcomes in patients treated with erlotinib for advanced chemotherapy-refractory NSCLC.

\section{MATERIALS AND METHODS}

\section{Patients and Eligibility}

This was a prospective study of serial ${ }^{11} \mathrm{C}-\mathrm{PD} 153035 \mathrm{PET} / \mathrm{CT}$ before and during treatment in patients receiving erlotinib for advanced NSCLC refractory to chemotherapy. Eligible patients had to have biopsy-proven NSCLC and progression on at least 1 chemotherapy regimen documented by CT. Every patient had to provide written consent to participate in this study, which was approved by the Institutional Review Board and the ethics committee at Shandong Cancer Hospital.

\section{Treatment}

Patients received erlotinib at an oral dose of $150 \mathrm{mg}$ daily. Treatment continued until disease progression or the advent of intolerable adverse effects.

\section{PET/CT}

Automated synthesis and ${ }^{11} \mathrm{C}$-radiolabeling of PD153035 from its precursor $(\mathrm{ABX} \mathrm{GmbH})$ were performed with a TRACERlab FX C system (GE Healthcare) and prepared for human use as described previously $(10,11)$. Baseline ${ }^{11} \mathrm{C}-\mathrm{PD} 153035 \mathrm{PET} / \mathrm{CT}$ was performed within $1 \mathrm{wk}$ before the initiation of treatment, and follow-up ${ }^{11} \mathrm{C}-\mathrm{PD} 153035$ PET/CT was performed at 1-2 wk and $6 \mathrm{wk}$ after the start of treatment. The time of $6 \mathrm{wk}$ was chosen because radiographic responses by CT to EGFR-TKIs are often evident by that time. Combined PET/CT scans were obtained with the patient supine using a PET/CT scanner (Discovery LS; GE Healthcare) capable of multislice helical CT for anatomic imaging and attenuation correction. No specific dietary instructions were given to the patients before or after PET/CT.

A dynamic emission acquisition sequence was started $20 \mathrm{~min}$ after the intravenous injection of $280.3 \pm 113.6 \mathrm{MBq}$ of ${ }^{11} \mathrm{C}$ PD153035. Images were acquired for a total of $3 \mathrm{~min}$ at each of 2 bed positions covering the neck and thorax. The time point of 20 min after injection was based on an unpublished observation from a prior pilot human study (11) that tumor uptake tended to be stable after $20 \mathrm{~min}$. The abdomen was excluded because the prior human study demonstrated diffuse high uptake in the abdomen (11). PET data were reconstructed on a $128 \times 128$ matrix (slice thickness, $4.25 \mathrm{~mm}$ ) using the ordered-subsets expectation maximization algorithm with 8 iterations and 4 subsets.

For standardized uptake value (SUV) analysis, ${ }^{11} \mathrm{C}$-PD153035 dynamic PET data at each bed position were summed over the total 3-min acquisition time to obtain static images of higher signal-to-noise ratio. The image slice with the maximum tumoral SUV was averaged with the 2 adjacent axial slices (1 plane above and 1 plane below the chosen slice) to improve count statistics, and the maximum value $\left(\mathrm{SUV}_{\max }\right)$ within the tumor region of interest was scored for each patient. SUV was calculated using the following formula: (measured activity concentration $[\mathrm{Bq} / \mathrm{mL}] \times$ body weight $[\mathrm{g}]) /$ injected activity $(\mathrm{Bq})$.

\section{Response Evaluation}

Contrast CT scans for treatment monitoring were acquired in all patients within $1 \mathrm{wk}$ before and at 6 -wk intervals after the start of the treatment, or sooner if indicated by clinical progression. An experienced radiologist who was unaware of the clinical course of patients interpreted each study, and response scoring was based on the response evaluation criteria in solid tumors (12). The best response was recorded for each patient.

\section{Statistical Analysis}

Overall survival and progression-free survival (OS and PFS, respectively) times were measured from the start of the treatment to the date of death or progression based on CT. Patients who were alive at the date of the last follow-up were censored for OS on that date. Patients who died without progressive disease documented were considered to have had progressive disease at the time of death. The correlation between ${ }^{11} \mathrm{C}$-PD153035 SUV and survival was analyzed by Cox regression analysis (13), and actuarial survival estimates were calculated according to the Kaplan-Meier method (14). With the Cox regression analysis, the SUV covariate was analyzed as a continuous linear variable, and with the Kaplan-Meier analysis, SUV was dichotomized into 2 groups separated by the median SUV. Before entering a variable into the Cox model, the proportional hazards assumption was assessed graphically by evaluating the log-minus-log survival plot. The baseline Cox regression model for OS and PFS included only the SUV covariate. To assess the effect of potential confounding covariates, we constructed Cox models with SUV and the potential confounders. The correlation between ${ }^{11} \mathrm{C}-\mathrm{PD} 153035$ SUV at different time points was assessed with a Pearson correlation coefficient. All statistical tests were 2 -sided, and $P$ values less than 0.05 were considered significant. Statistical analysis was performed using SAS software (version 9.2; SAS Institute Inc.).

TABLE 1

Patient Characteristics

\begin{tabular}{|c|c|c|}
\hline Characteristic & $\begin{array}{c}\text { No. of } \\
\text { patients } \\
(n=21)\end{array}$ & Percentage \\
\hline \multicolumn{3}{|l|}{ Sex } \\
\hline Male & 8 & 38 \\
\hline Female & 13 & 62 \\
\hline \multicolumn{3}{|l|}{ Tumor stage } \\
\hline IIIB & 1 & 5 \\
\hline IV & 20 & 95 \\
\hline \multicolumn{3}{|l|}{ Histopathologic subtype } \\
\hline Adenocarcinoma & 15 & 71 \\
\hline Squamous cell carcinoma & 6 & 29 \\
\hline \multicolumn{3}{|l|}{ Smoking history } \\
\hline Current & 1 & 5 \\
\hline Former & 9 & 43 \\
\hline None & 11 & 52 \\
\hline \multicolumn{3}{|l|}{ Prior cisplatin } \\
\hline Yes & 21 & 100 \\
\hline No & 0 & 0 \\
\hline \multicolumn{3}{|l|}{ No. of prior treatment regimens } \\
\hline 1 & 6 & 29 \\
\hline 2 or 3 & 15 & 71 \\
\hline \multicolumn{3}{|c|}{ Best response to prior chemotherapy } \\
\hline Complete or partial response & 7 & 33 \\
\hline Stable disease & 10 & 48 \\
\hline Progression & 4 & 19 \\
\hline
\end{tabular}

Median age of patients was 62 y (range, 39-79 y). 


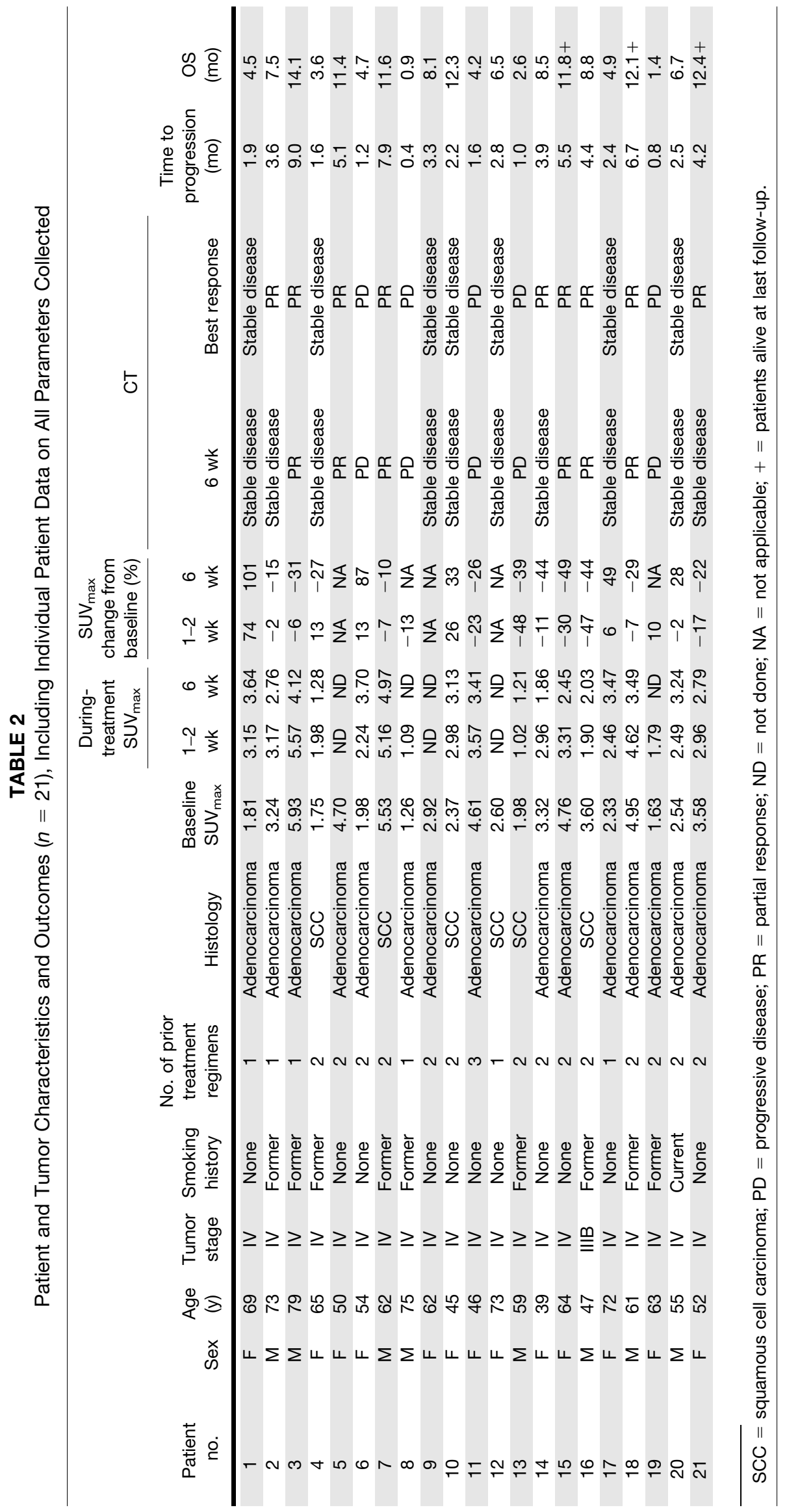




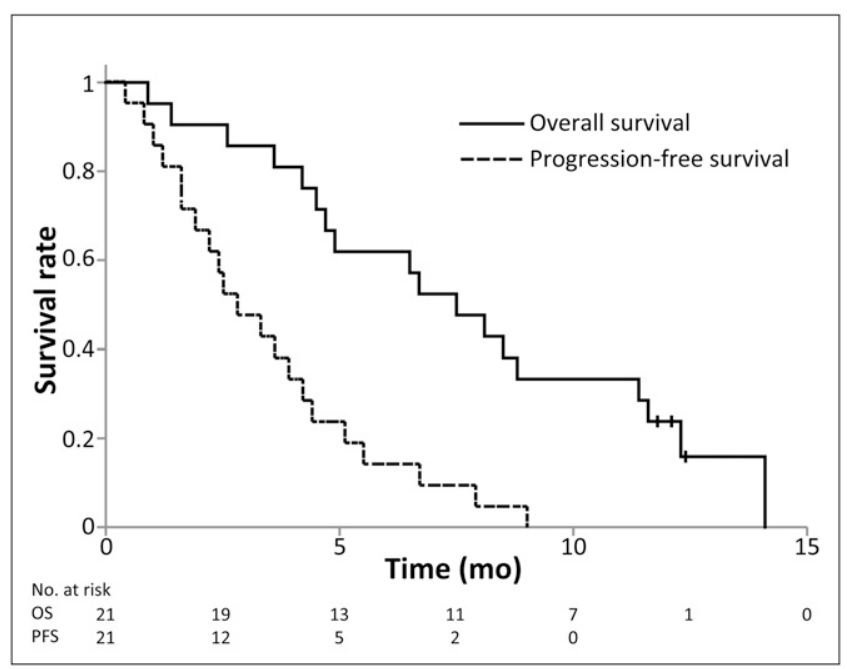

FIGURE 1. Kaplan-Meier OS and PFS curves for entire cohort of 21 patients with advanced chemotherapy-refractory NSCLC treated with erlotinib.

\section{RESULTS}

\section{Patients and Overall Outcomes}

Twenty-one patients were enrolled at Shandong Cancer Hospital for the ${ }^{11} \mathrm{C}-\mathrm{PD} 153035 \mathrm{PET} / \mathrm{CT}$ study between June 2008 and May 2009. Their characteristics are summarized in Table 1. All patients had biopsy-proven adenocarcinoma or squamous cell carcinoma. However, genotyping for EGFR mutation status, immunohistochemistry for EGFR protein expression, and EGFR gene copy number determination were not routinely performed and therefore not available for analysis. Follow-up to progression was complete in all patients, and all patients progressed before death. At the time of this analysis, 18 of 21 patients had died. Median follow-up time was 7.5 mo.

All patients were treated with oral erlotinib per the protocol. Three patients also received radiation therapy for palliation of osseous metastases during the course of the study. The individual survival outcomes are detailed in Table 2, along with all parameters recorded in the study. Four patients (patients 6, 8, 13, and 19) had clinical progression and received CT scans before the scheduled 6-wk evaluation time point. Two patients (patients 1 and 4) progressed clinically shortly after the 6-wk CT documented stable disease and were scored as having progression at the tabulated time points.

Figure 1 shows the Kaplan-Meier estimated OS and PFS for the cohort. The median OS and PFS were 7.5 and 2.8 mo, respectively. The 6-mo actuarial OS and PFS rates were $62 \%$ and $14 \%$, respectively.

\section{${ }^{11} \mathrm{C}-\mathrm{PD} 153035$ PET/CT and Correlation with Outcomes}

All patients underwent baseline ${ }^{11} \mathrm{C}-\mathrm{PD} 153035 \mathrm{PET} / \mathrm{CT}$. Three patients underwent only the baseline scan. An additional 2 patients did not undergo the third scan because of clinical deterioration. Therefore, ${ }^{11} \mathrm{C}-\mathrm{PD} 153035 \mathrm{PET} / \mathrm{CT}$

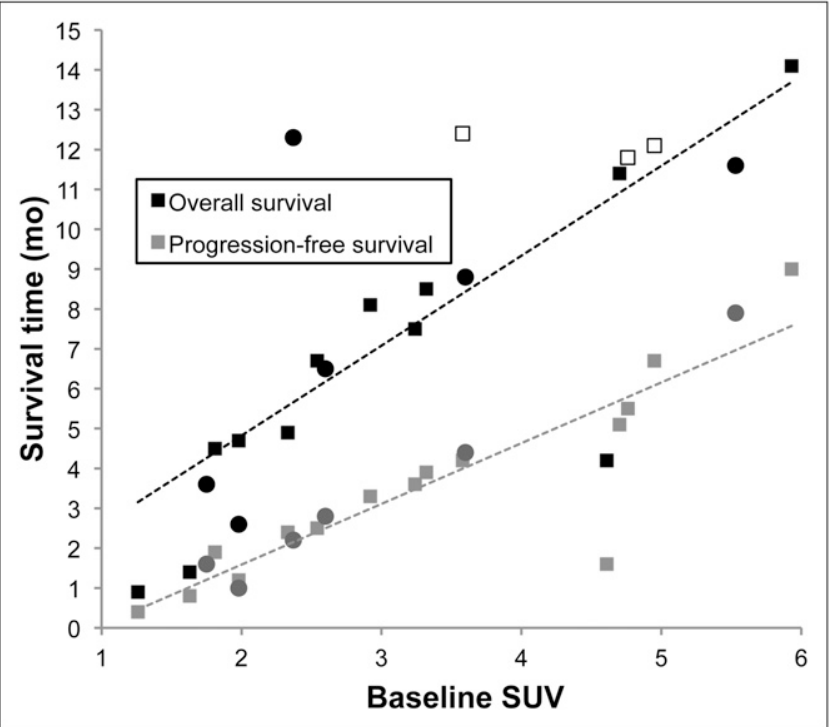

FIGURE 2. Plots of survival times by baseline ${ }^{11} \mathrm{C}$-PD153035 $S_{\text {SUV }}$ max. Both OS and PFS times correlated strongly with uptake of tracer. Relationships between SUV and survival times appear to hold independently of tumor histology. Squares indicate adenocarcinoma, and circles squamous cell carcinoma. $\square=$ censored times.

after 1-2 wk was available in 18 patients and after $6 \mathrm{wk}$ in 16 patients. The baseline ${ }^{11} \mathrm{C}-\mathrm{PD} 153035 \mathrm{PET} / \mathrm{CT}$ was performed $1.4 \pm 0.9 \mathrm{~d}$ before the start of treatment, and follow-up scans were obtained at $10.8 \pm 3.1 \mathrm{~d}(1.4 \pm 0.5$ wk) and $43.7 \pm 2.9 \mathrm{~d}(6.0 \pm 0.4 \mathrm{wk})$ after the start of treatment. The $\mathrm{SUV}_{\max }$ at each time point is detailed in Table 2, as are the relative changes between the duringtreatment and baseline scans.

Baseline ${ }^{11} \mathrm{C}-\mathrm{PD} 153035 \mathrm{SUV}_{\max }$ correlated strongly and highly significantly with OS and PFS times (Fig. 2). On Cox regression analysis, each unit increase in $\mathrm{SUV}_{\max }$ reduced the hazard of death by $60 \%$ (hazard ratio $[\mathrm{HR}]=$ $0.40 ; 95 \%$ confidence interval $[\mathrm{CI}]=0.22-0.70 ; P=0.002$ ) and reduced the hazard of progression by $96 \%$ (HR $=0.044$; 95\% CI $=0.01-0.22 ; P<0.001$ ) (also apparent visually in Fig. 2). Figure 3 shows an example of a patient with a high baseline $\mathrm{SUV}_{\max }$ who had a good response by CT to erlotinib and remained alive at nearly 12 mo of follow-up.

${ }^{11} \mathrm{C}-\mathrm{PD} 153035 \mathrm{SUV}_{\max }$ early in treatment (at $1-2 \mathrm{wk}$ ) also correlated with OS and PFS, with an HR of 0.36 (95\% $\mathrm{CI}=0.17-0.75 ; P=0.007)$ and an HR of $0.29(95 \% \mathrm{CI}=$ $0.14-0.60 ; P=0.001)$, respectively. $\mathrm{SUV}_{\max }$ at $1-2 \mathrm{wk}$ correlated strongly with baseline $\mathrm{SUV}_{\max }(r=0.87$ [95\% $\left.\mathrm{CI}=0.65-0.95], R^{2}=0.75, P<0.0001\right)$.

On the other hand, SUV $_{\max }$ at about 6 wk did not correlate with baseline $\mathrm{SUV}_{\max }(r=0.47$ [95\% CI $=-0.05-$ $0.78], R^{2}=0.22, P=0.066$ ), and was not associated with OS (HR $=0.66 ; P=0.25)$ or PFS (HR $=0.055 ; P=$ 0.066) on Cox regression analysis. The absolute change in $\mathrm{SUV}_{\max }$ between $6 \mathrm{wk}$ and baseline was inversely correlated with baseline $\mathrm{SUV}_{\text {max }}(r=-0.71[95 \% \mathrm{CI}=-0.89$ to -0.31$\left.], R^{2}=0.51, P=0.001\right)$, reflecting that initially 

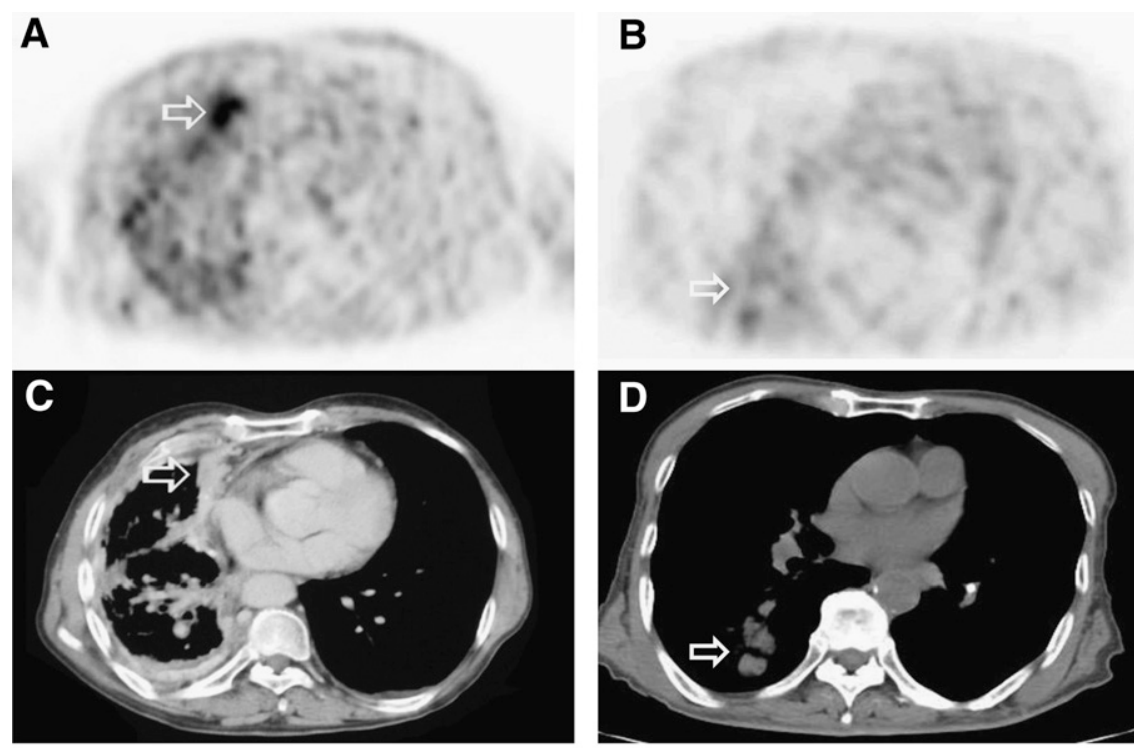

FIGURE 3. Baseline ${ }^{11} \mathrm{C}-\mathrm{PD} 153035$ PET before erlotinib treatment in patient 15 (A) with adenocarcinoma and patient 13 (B) with squamous cell carcinoma. Arrows indicate lesions with highest $S U V_{\text {max }}$ of 4.76 and 1.98 in patients 15 and 13 , respectively. Corresponding CT slices from before treat-
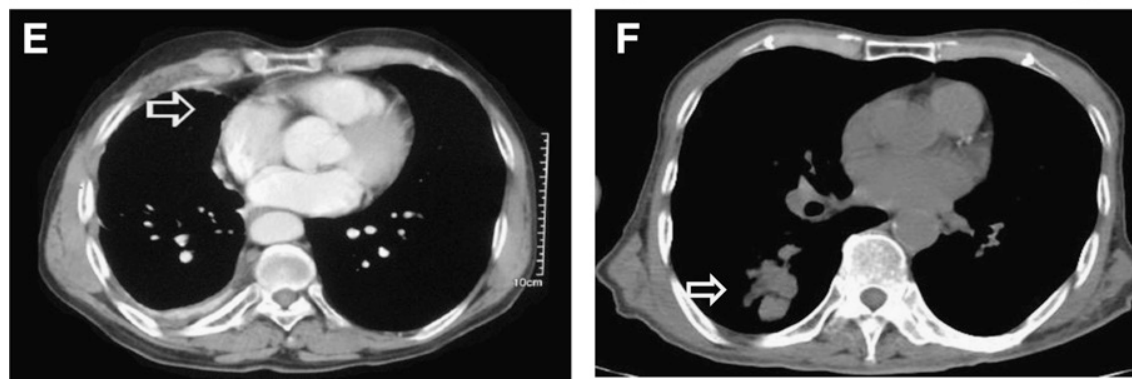
ment $(C$ and $D)$ and 6 wk after treatment ( $E$ and $F$ ). Patient 15, with higher baseline $\mathrm{SUV}_{\max }$, had marked radiographic improvement by 6 wk after erlotinib was initiated (A, C, and E). Tumor progression ultimately occurred at $5.5 \mathrm{mo}$, and patient remained alive at last follow-up of 11.8 mo. However, patient 13, with lower baseline SUV $V_{\max }$, had radiographic progression 6 wk after erlotinib was initiated $(B, D$, and $F)$ and died within $2.6 \mathrm{mo}$.

low SUVs tended to increase by 6 wk whereas initially high SUVs tended to decrease.

For the Kaplan-Meier survival analysis, patients were stratified by greater or less than the median baseline ${ }^{11} \mathrm{C}$ PD153035 SUV max $_{\text {of }} 2.92$ (Fig. 4). Patients with a baseline $\mathrm{SUV}_{\max }$ of 2.92 or greater had significantly longer median OS (11.4 vs. 4.6 mo) and higher 6-mo OS (91\% vs. 30\%) than did patients with an $\mathrm{SUV}_{\max }$ of less than $2.92(P=$ 0.002). Similarly, median PFS (4.4 vs. $1.8 \mathrm{mo}$ ) and 6-mo PFS (27\% vs. $0 \%$ ) were significantly better with high versus low $\mathrm{SUV}_{\text {max }}(P<0.001)$.

\section{Other Factors}

We assessed the role of potential confounders and their effect on the association between baseline ${ }^{11} \mathrm{C}$-PD153035 $\mathrm{SUV}_{\text {max }}$ and outcome (Supplemental Table 1; supplemental materials are available online only at http://jnm.snmjournals. org online only). After controlling for histology, baseline ${ }^{11} \mathrm{C}-\mathrm{PD} 153035 \mathrm{SUV}_{\max }$ continued to predict $\mathrm{OS}(\mathrm{HR}=$ $0.36, P=0.002)$ and PFS $(\mathrm{HR}=0.043, P<0.001)$. After adjusting for smoking history (current or former vs. none), baseline ${ }^{11} \mathrm{C}-\mathrm{PD} 153035 \mathrm{SUV}_{\max }$ continued to predict $\mathrm{OS}(\mathrm{HR}=0.31, P=0.002)$ and PFS $(\mathrm{HR}=0.079$, $P<0.001)$. Finally, after controlling for the number of prior chemotherapy regimens (1 vs. $2-3$ ), baseline ${ }^{11} \mathrm{C}$ PD153035 SUV $\max$ continued to predict OS $(\mathrm{HR}=0.42$, $P=0.004)$ and PFS (HR $=0.076, P<0.001)$. That the HRs for OS and PFS remain relatively unchanged after adjusting for each of these parameters suggests that they were unlikely to confound the relationship between baseline ${ }^{11} \mathrm{C}$-PD153035 $\mathrm{SUV}_{\max }$ and outcome.

\section{DISCUSSION}

This prospective study provides the first, to our knowledge, evidence that ${ }^{11} \mathrm{C}$-PD153035 PET/CT can predict outcomes in patients treated with EGFR-TKI for advanced chemotherapy-refractory NSCLC. Several of the findings in this study are notable.

The patient population in this study is similar to those of the INTEREST and NCIC BR.21 trials in comprising patients with advanced chemotherapy-refractory NSCLC, albeit with a greater proportion of patients who received 2 or more prior chemotherapy regimens (71\% vs. 16\%-49\%) but who also had a higher proportion of adenocarcinoma (71\% vs. $50 \%-54 \%$ ), were never smokers (52\% vs. $20 \%$ ), and were of Asian ethnicity (100\% vs. 19\%-22\%) (5,6). We observed a median OS comparable to those in the EGFRTKI arms of those trials (7.5 mo vs. 6.7-7.6 mo).

When stratified by above or below the median baseline ${ }^{11} \mathrm{C}-\mathrm{PD} 153035 \mathrm{SUV}_{\max }$ of 2.92, the highly significant difference between median OS of 11.4 versus 4.6 mo appears similar in magnitude to the impact of EGFR molecular markers in the EGFR-TKI treatment arms of the BR.21 and INTEREST trials (median OS with vs. without the presence of the markers $=\sim 9-14$ mo vs. $\sim 6 \mathrm{mo})(7,8)$. 


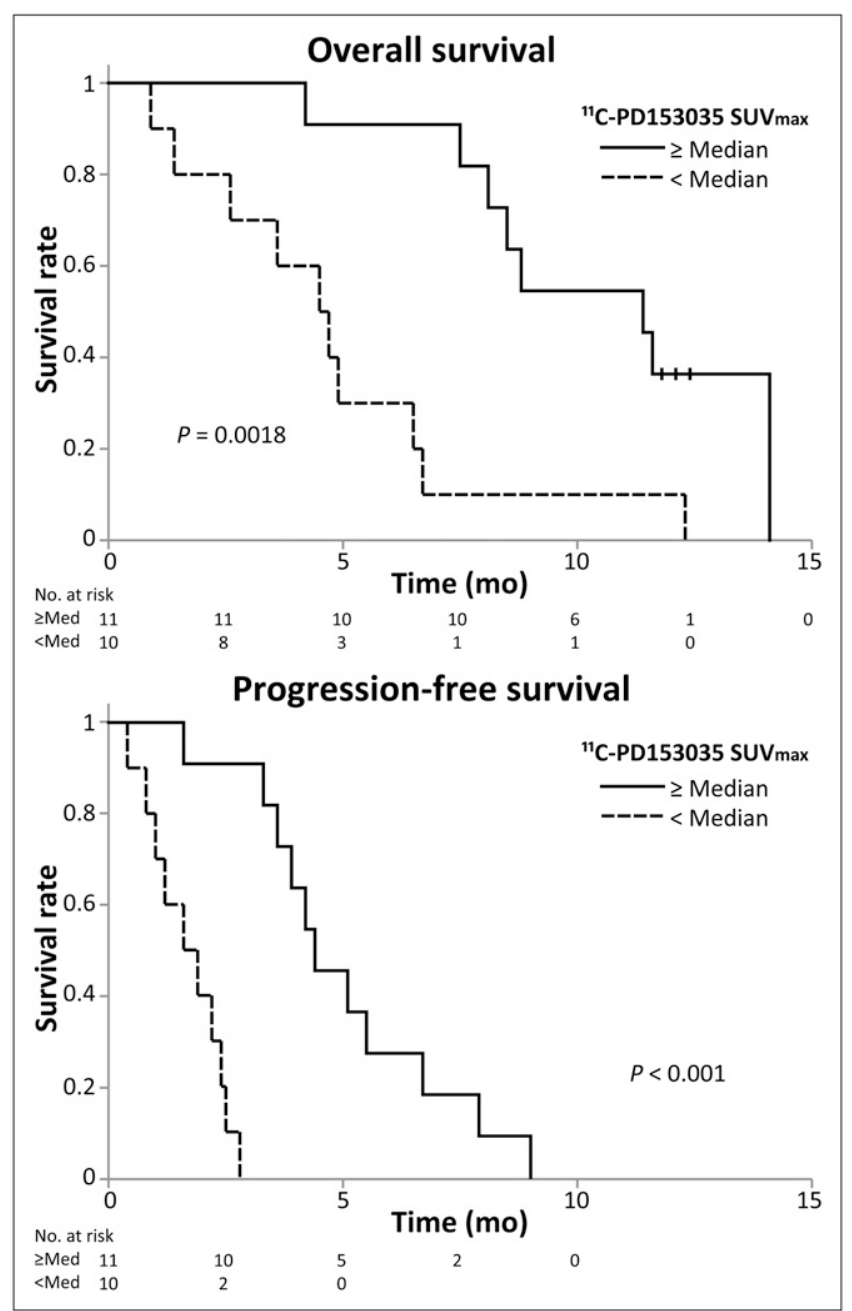

FIGURE 4. Kaplan-Meier OS and PFS curves stratified by baseline ${ }^{11} \mathrm{C}$-PD153035 SUV $\max$ greater or less than median value of 2.92. Differences between survivals in high and low SUV groups were highly significant.

However, those 2 trials had disparate findings regarding which molecular markers were predictive: EGFR expression and copy number but not mutation status in BR.21 $(7,8)$ and vice versa in INTEREST (but not reaching statistical significance between treatment arms) (8). As an imaging biomarker, ${ }^{11} \mathrm{C}-\mathrm{PD} 153035 \mathrm{PET} / \mathrm{CT}$ has the advantage of being noninvasive and thus potentially more widely applicable and should be less susceptible to sampling inadequacy because it provides an assessment of the entire tumor volume.

These advantages of ${ }^{11} \mathrm{C}$-PD153035 PET/CT raise the question of whether this biomarker correlates with one or more of the known EGFR tissue biomarkers and whether it provides independent information. An unfortunate limitation of this study was the inability to obtain the tissue biomarkers for comparison to imaging. In a preclinical study, uptake of ${ }^{11} \mathrm{C}$-PD153035 in different cell lines and their corresponding xenografts correlated strongly with the level of EGFR protein expression (10). A preclinical study of a related PET tracer, ${ }^{11} \mathrm{C}$-erlotinib, found that, similarly, the uptake was highest in the cell line with the highest EGFR expression, but this was also the cell line that harbored an activating mutation in EGFR (15). Thus, uptake of radiolabeled EGFR-TKIs might correlate with more than one EGFR biomarker.

Another interesting observation in this study was that the relationship of outcomes with ${ }^{11} \mathrm{C}$-PD153035 PET/CT in adenocarcinomas appeared no different from that in squamous cell carcinomas, which rarely harbor the common EGFR-activating mutations (16) as reflected by the unchanged HRs with and without adjustment for histology. Although the number of patients was too small to make strong conclusions, this observation would suggest that this imaging biomarker may provide information complementary to the tissue biomarkers of EGFR and have value in a broader patient population.

Finally, serial imaging with ${ }^{11} \mathrm{C}-\mathrm{PD} 153035 \mathrm{PET} / \mathrm{CT}$ revealed that the later follow-up time point (at $\sim 6$ wk after the start of erlotinib therapy) yielded no useful prognostic information beyond what was provided by the baseline scan. The strong correlation between the early follow-up scan and the baseline scan suggests that there is a time window of about 1-2 wk around the start of therapy within which ${ }^{11} \mathrm{C}-\mathrm{PD} 153035$ PET/CT may be useful for outcome prediction. Zander et al. (17) and Sohn et al. (18) found that ${ }^{18} \mathrm{~F}-\mathrm{FDG}$ PET and ${ }^{18} \mathrm{~F}$-FLT PET, respectively, early in the course of treatment could predict outcomes to EGFR-TKI treatment. An advantage of ${ }^{11} \mathrm{C}$-PD153035 is the ability to predict response before the start of therapy.

Although we failed to see a correlation between outcome and ${ }^{11} \mathrm{C}-\mathrm{PD} 153035 \mathrm{SUV}_{\max }$ at $6 \mathrm{wk}$, fewer patients underwent scans at later time points, reducing the power to detect a correlation if one in fact existed. Additionally, a more robust method of analyzing the data would have been to use time-dependent models, which would account for the within-person correlation of SUV at different time points; however, the small sample size limited our ability to use this approach.

\section{CONCLUSION}

The promising results of this study justify a larger trial of ${ }^{11} \mathrm{C}-\mathrm{PD} 153035 \mathrm{PET} / \mathrm{CT}$ in populations of patients receiving EGFR-TKIs for NSCLC that includes correlation with EGFR tissue biomarkers.

\section{DISCLOSURE STATEMENT}

The costs of publication of this article were defrayed in part by the payment of page charges. Therefore, and solely to indicate this fact, this article is hereby marked "advertisement" in accordance with 18 USC section 1734.

\section{ACKNOWLEDGMENTS}

We thank the staff of the PET/CT Center, Shandong Cancer Hospital, particularly Shuqiang Zhao, Lusheng 
Chen, Zheng Fu, Xuepeng Teng, and Jinsong Zheng, for their technical support. This work was supported by the National High Technology Research and Development Program of China (grant 2007 AA02Z437) and the National Natural Science Foundation (30870748). No other potential conflict of interest relevant to this article was reported.

\section{REFERENCES}

1. Jemal A, Bray F, Center MM, et al. Global cancer statistics. CA Cancer J Clin. 2011;61:69-90.

2. Schiller JH, Harrington D, Belani CP, et al. Comparison of four chemotherapy regimens for advanced non-small-cell lung cancer. N Engl J Med. 2002;346:92-98.

3. Scagliotti GV, De Marinis F, Rinaldi M, et al. Phase III randomized trial comparing three platinum-based doublets in advanced non-small-cell lung cancer. $J$ Clin Oncol. 2002;20:4285-4291.

4. Mok TS, Wu Y-L, Thongprasert S, et al. Gefitinib or carboplatin-paclitaxel in pulmonary adenocarcinoma. $N$ Engl J Med. 2009;361:947-957.

5. Shepherd FA, Rodrigues Pereira J, Ciuleanu T, et al. Erlotinib in previously treated non-small-cell lung cancer. $N$ Engl J Med. 2005;353:123-132.

6. Kim ES, Hirsh V, Mok T, et al. Gefitinib versus docetaxel in previously treated non-small-cell lung cancer (INTEREST): a randomised phase III trial. Lancet. 2008;372:1809-1818.

7. Tsao M-S, Sakurada A, Cutz J-C, et al. Erlotinib in lung cancer: molecular and clinical predictors of outcome. N Engl J Med. 2005;353:133-144.

8. Douillard J-Y, Shepherd FA, Hirsh V, et al. Molecular predictors of outcome with gefitinib and docetaxel in previously treated non-small-cell lung cancer: data from the randomized phase III INTEREST trial. J Clin Oncol. 2010;28:744-752.
9. Fredriksson A, Johnström P, Thorell JO, et al. In vivo evaluation of the biodistribution of ${ }^{11} \mathrm{C}$-labeled PD153035 in rats without and with neuroblastoma implants. Life Sci. 1999;65:165-174.

10. Wang H, Yu J, Yang G, et al. Assessment of ${ }^{11} \mathrm{C}$-labeled-4- $N$-(3-bromoanilino)6,7-dimethoxyquinazoline as a positron emission tomography agent to monitor epidermal growth factor receptor expression. Cancer Sci. 2007;98:1413-1416.

11. Liu N, Li M, Li X, et al. PET-based biodistribution and radiation dosimetry of epidermal growth factor receptor-selective tracer ${ }^{11} \mathrm{C}$-PD153035 in humans. J Nucl Med. 2009;50:303-308.

12. Therasse P, Arbuck SG, Eisenhauer EA, et al. New guidelines to evaluate the response to treatment in solid tumors. European Organization for Research and Treatment of Cancer, National Cancer Institute of the United States, National Cancer Institute of Canada. J Natl Cancer Inst. 2000;92:205-216.

13. Cox D. Regression models and life-tables. J R Stat Soc, B. 1972;34:187-220.

14. Kaplan E, Meier P. Nonparametric estimation from incomplete observations. J Am Stat Assoc. 1958;53:457-481.

15. Memon AA, Jakobsen S, Dagnaes-Hansen F, et al. Positron emission tomography $(\mathrm{PET})$ imaging with $\left[{ }^{11} \mathrm{C}\right]$-labeled erlotinib: a micro-PET study on mice with lung tumor xenografts. Cancer Res. 2009;69:873-878.

16. Marchetti A, Martella C, Felicioni L, et al. EGFR mutations in non-small-cell lung cancer: analysis of a large series of cases and development of a rapid and sensitive method for diagnostic screening with potential implications on pharmacologic treatment. J Clin Oncol. 2005;23:857-865.

17. Zander T, Scheffler M, Nogova L, et al. Early prediction of nonprogression in advanced non-small-cell lung cancer treated with erlotinib by using ${ }^{18} \mathrm{~F}$-fluorodeoxyglucose and ${ }^{18} \mathrm{~F}$-fluorothymidine positron emission tomography. J Clin Oncol. 2011;29:1701-1708.

18. Sohn HJ, Yang YJ, Ryu JS, et al. ${ }^{18}$ F-fluorothymidine positron emission tomography before and 7 days after gefitinib treatment predicts response in patients with advanced adenocarcinoma of the lung. Clin Cancer Res. 2008;14:74237429 . 